\title{
Optimized Handover Schemes over WiMAX
}

\author{
Zina Jerjees, H.S. Al-Raweshidy, and Zaineb Al-Banna \\ Brunel University, Wireless Networks and Communications Centre (WNCC), \\ London, UB8 3PH, UK \\ \{Zina.Jerjees, Zaineb.Al-Banna\} @brunel.ac.uk
}

\begin{abstract}
Voice Over Internet Protocol (VoIP) applications have received significant interests from the Mobile WiMAX standard in terms of capabilities and means of delivery multimedia services, by providing high bandwidth over longrange transmission. However, one of the main problems of IEEE 802.16 is that it covers multi BS with too many profiled layers, which can lead to potential interoperability problems. The multi BS mode requires multiple BSs to be scanned synchronously before initiating the transmission of broadcast data. In this paper, we first identify the key issues for VoIP over WiMAX. Then we present a MAC Layer solution to guarantee the demanded bandwidth and supporting a higher possible throughput between two WiMAX end points during the handover. Moreover, we propose a PHY and MAC layers scheme to maintain the required communication channel quality for VoIP during handover. Results show that our proposed schemes can significantly improve the network throughput up to 55\%, reducing the data dropped to $70 \%$ while satisfying VoIP quality requirements.
\end{abstract}

Keywords: WiMAX, handover, cross-layer.

\section{Introduction}

The IEEE 802.16 standard has emerged as an important technology for delivering packet data service in a wide area cellular network [1] [2]. As multi-homed WiMAX will characterize future wireless systems investigating the development of intelligent and efficient handover management mechanisms that can provide seamless roaming capability to mobile users moving between several different access networks, providing a high bandwidth for high speed applications and reduce the overhead cost. However, a traditional problem may occur in the standard, is that it covers too many profiles and PHY layers [3], which can lead to potential interoperability problems as the mobile user traverse between large number of access devices. In ubiquitous networks, mobile nodes can traverse hotspots while maintaining Voice over Internet Protocol (VoIP) communication. Thus, a single VoIP communication can experience several handovers, which can lead to packet loss, resulting in the deterioration of VoIP communication quality. On the other hand, the VoIP different streams may follow the same path through between WiMAX two end points, the Mobile Station (MS) and the base station (BS). This means that the capacity (bandwidth) of that path (defined by its Link layer) severely restricts the amount of critical video streams that can be viewed in emergency situations considering Quality of Service (QoS) parameters. Therefore, preventing packet losses 
during handover is crucial for high-quality VoIP communication. Thus, enhance us to introduce our handover proposals in order to guarantee VoIP minimum requirements. Since the Mobile WiMAX also provides high performance due to relying upon the upgraded PHY layer Orthogonal Frequency Division Multiplexing Access (OFDMA) which, as multiplexing technique, supports multipath environments, thus resulting in the ability to generate higher throughput and improved network coverage. The MAC handover process introduces a large number of interactions between the MS and adjacent (neighboring) BSs for the purpose of scanning, ranging, parameter negotiation and information exchanging.

Our first proposed solution, to improve the mobile WiMAX handover and address the latency issue. The second solution is to adapt the channel congestion problem and how it severely restricts the amount of critical video streams, introducing an algorithm that based on the channel bandwidth information, which effectively reduce the mobile WiMAX handover (HO) latency.

In section 2 of this paper, background and related work with an overview of WiMAX MAC layer and handover process moving forward to discuss the handover performance requirements, showing different levels of services for providing WiMAX QoS. Then in section 3 we present our proposed schemes to improve the throughput and traffic loss issues and reduce the handover latency. Section 4 presents simulations network model, section 5 presents the performed results and discussion, and finally section 6 concludes the paper.

\section{Background and Related Work}

\subsection{Related Work}

A handover solution in [4] proposes a Link-layer handover algorithm that enhances the ranging of data streams between the serving BS and the neighboring BSs, thus enables the MS to receive the data downstream at the time it becomes synchronized with the neighboring BSs, the limitation of this scheme to downstream only from BS to MS may lead to the deterioration of VoIP communication quality as it requires a two directions streams (down and upstream) handover enhanced solution. The proposal in [5] introduce a cross-layer approach for fast handover, the concept relies on the use of an extended MAC-layer information being sent to every channel between the MS and the BSs, by including the IP address information to the MAC probingmessages, as this reduces the time to scan all available channels. The drawbacks of this scheme, the availability of the MS IP address in this open way may grant the chance to an unsecure access device to obtain that address just simply. The other drawback is the buffering of the probe message by an access device for a certain fixed time before it sends it out may cause an additional delay for the MS to connect to its communicating channel. [6] Through the backbone message, a BS sends information to neighbor BSs. The MS selects candidate target BS based on the signal strength and response time of that $\mathrm{BS}$.

Our proposals present a fast handover scheme which reduces HO latency in mobile WiMAX scenarios. The MS will follow the scanning/ranging steps with eliminated number of BSs. As the VoIP quality will be degraded for a reason or another, our 
schemes will try to solve some channel issues such the traffic congestion and traffic routing without affecting the $\mathrm{HO}$ prospects as in the other proposed schemes.

\subsection{IEEE 802.16 MAC Layer Handover Support}

In IEEE 802.16e standard, three kinds of handover types are supported [10]:

1. Hard handover (HHO)

2. Fast BS switching (FBSS) and

3. Macro Diversity Handover (MDHO)

Among these handover types, HHO is the simplest one while the other two types are more complicated and optional. Up till now, only HHO and FBSS are adequately defined in the standard for practical use. However, both had the physical radio link broken before it is re-established at the target access point that results in handshake latency. In this paper, when handover is mentioned, it refers to HHO. The handover process in WiMAX is concluded in the movement of the MS from a BS to another BS with a connection to a different air interface to provide the desired communication requirements.

The WiMAX handover procedure is consisting of several scenarios as shown in the following section for its all-over acquaintance [7] [11].

\subsubsection{Network Topology Advertisement}

The BSs periodically broadcast Mobile Neighbor Advertisement (MOB_NBR_ADV) control messages which contain both physical layer (i.e., radio channel) and link layer (e.g., MAC address) information, Bandwidth and QoS. By means of such broadcasts, the MS becomes aware of the neighboring BSs. The MS then triggers the scan-phase.

\subsubsection{Scanning/Ranging Procedure}

In the scan phase of $\mathrm{HO}$, the MS scans and synchronizes with the neighboring BSs based on channel information from the neighbor advertisement. In order to find an appropriate BS target, the serving BS scans the available channels of the down-link (DL) frequency band. The MS is looking for a well-known DL preamble frame [11]. Since the preamble is transmitted periodically, the BS should gather the frame duration and having synchronized it on time. As a result of receiving preamble frame; the channel estimation, initialization and equalization procedures are taken place. The Frame Control Header (FCH) or the Down Link Frame Prefix (DLFP) which are following the DL preamble can be decoded to the DL burst, which contains the DL mapping message (DL MAP) and the Downlink Channel Descriptor (DCD). These burst messages contain all the required parameters for enabling the DL transmission. When successfully the DL MAP and the DCD are being received, the MAC and the PHY synchronization of the BS is started. If the synchronization successes, it then starts the ranging procedure. The scanning and ranging processes are shown in Figure 1. the BS decodes the Uplink Channel Descriptor (UCD) that contains the uplink centre frequency and the size of the initial ranging contention slots in order to obtain the required uplink parameters, as the BS may need to correct its offset frequency timers and adjusts its transmission power. The MS regularly receives channel information about the neighboring BSs through the MOB_NBR-ADV message from 
the current serving BS. If the signal strength weakens, The MS will send a Mobile Scanning Request (MOB_SCN_REQ) message to the neighboring BS with a potential target BS list (selected in the previous phase). The serving BS replies a Mobile Scanning Response (MOB_SCN-RSP) message to the MS to allocate a scanning duration. The MS selects candidate target BSs based on the signal strength and response time of each BS, acquired from scanning. The serving BS may negotiate directly with the listed BSs the allocation of a unicast ranging opportunity. If successful, the ranging procedure can be non-contention-based.

Else, the MS starts a contention-based CDMA procedure to be allocated a ranging slot by the neighboring BS. For the sake of simplicity, in analysis we always assume non-contention-based-ranging. Then the MS starts a hand-shake ranging procedure with the neighboring BS for the OFDMA uplink synchronization and parameter (e.g., transmission power) adjustment. The MS starts choosing an initial ranging contention slot to send its ranging messages (Ranging Request (RNG-REQ). These messages are addressed to the reserved CID. Then the neighboring BS responds with Ranging Response (RNG-RSP). This response message includes the primary management CID, transmission power information and the frequency timing offset adjustments. This procedure ends after the MS has completed ranging with all its neighbors. In the ranging phase, a MS may switch to a new channel, thus temporally loosing connection with the serving BS.

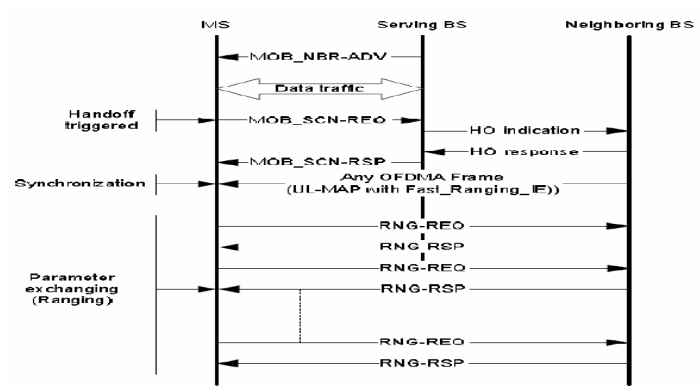

Fig. 1. Scanning and Ranging procedure

From the physical view; Synchronization of a MS across multi-BS is hard to achieve. The synchronization here means for an example; same video content is transmitted in the same OFDMA frame, the same OFDMA data region by the same channel coding scheme [11]. MS synchronization is critical not only for achieving macro-diversity and reducing interference, but also for smooth handover.

\subsubsection{HO Decision and Initiation}

The HO trigger decision and initiation can be originated by both the MS and the BS using a MS HO Request message (MOB_MSHO-REQ) or a BS HO Request message (MOB_BSHO-REQ) respectively. Here we use the HO started by the MS as an example as illustrated in Figure 2. The MS makes a decision about which BS(s) is (are) its target(s). A HO begins with when the MS sends a MOB_MSHO-REQ message to its serving BS indicating one or more possible target BSs. The serving BS may obtain directly from potential target BSs the expected MS performance at the target BSs through the 
exchange of $\mathrm{HO}$ indication and response messages. After receiving a response from a target BS (MOB_BSHORSP), the MS notifies the serving BS about its decision to perform a $\mathrm{HO}$ by means of a $\mathrm{HO}$ Indication (MOB_HO-IND) message. The MS can also ask the serving BS to negotiate with the target BS the allocation of a ranging opportunity. If necessary, the MS may start ranging after $\mathrm{HO}$ initiation. The $\mathrm{HO}$ decision and initiate process does not provoke connectivity break-up nor does it add latency. However, the possible ranging procedure after does introduce additional latency.

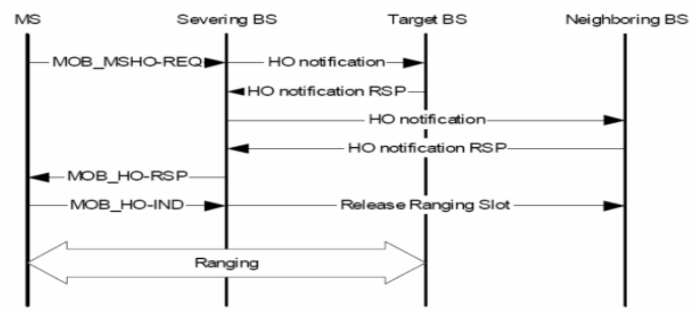

Fig. 2. Handover Decision and Initiation

\subsubsection{Network Re-entry}

After all the physical parameter adjustments have been completed successfully, the network re-entry process is initiated to establish connectivity between the MS and the target BS. As defined in IEEE 802.16e, this procedure may include capability negotiation, authentication and registration transactions. The capabilities negotiation includes information such like; the length of the Transmit/ Receive Transition Gaps (TTG) and (RTG) and how much the maximum transmission power. The authentication includes the exchange of encryption keys. And the registration is the last step before the MS is allowed to enter the network; the MS sends its registration request message that contains information about its Automatic Repeat Request (ARQ) and Cyclic Redundancy Check (CRC) and the MAC CS. the MS must wait until the re-entry procedure is completed successfully before it can restore communication. Then the MS has to manage its IP connectivity establishment to download the configuration file from its server. When this download is done, the network re-entry is completed. The duration of this phase should be taken account into the entire HO latency.

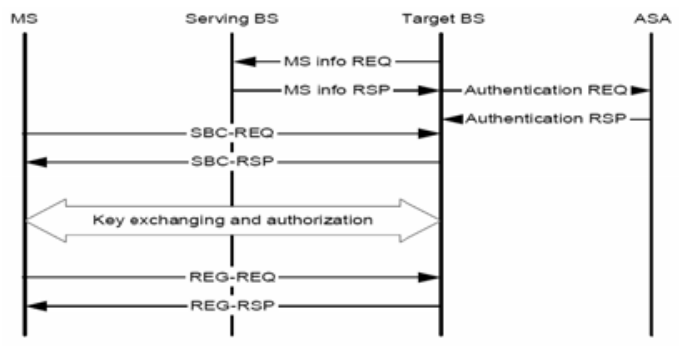

Fig. 3. Network Re-entry 


\subsection{Criterion Causes Handover}

All above procedures may affect the video broadcast performance over WiMAX. This motivates us to highlight some issues which may lead to handover decision in ubiquitous WiMAX:

1. Synchronization of MS across multi-BS is hard to achieve since the same application i.e., video stream content has to be transmitted in the same OFDMA frame and by the same channel usage. The MS synchronization is critical to achieve macrodiversity, reducing interference and reducing the handover. The reason why it is hard to achieve synchronization is as follows: different levels of delay to transmit video data packets copy from MS to BSs, as this video packet copy could also be lost during transmission on the channel between the MS and the BSs. Therefore the same OFDMA will not transmit the same video data packets.

2. There is a possibility of large-size video frame to be transmitted and share the medium. This will arise a congestion issue since the same channel will carry the same video data packets. Thus the video quality will degrade significantly when MS experience temporal fading or interference. In addition, the burst transmission mechanism that allows multiple MAC PDUs belonging to the same video channel to be exchanged in an aggregate way [9]. And if the burst size is big, this may cause an additional delay rate to the channel access operation.

Therefore, to achieve a seamless handover the following three requirements should be considered:

1. Prompt and reliable detection of degradation in wireless link (channel) quality between end-points (MS and BS).

2. Elimination of handover processing elements on Network (if possible), Link and Physical layers.

\section{Proposed Handover Solutions over WIMAX}

\subsection{Fast Channel Scanning (FCS) Based on Bandwidth Information}

The first proposed scheme to show that the scanning time is reduced in accordance with the number of eliminated BSs based on the negotiations procedure. Therefore the handover latency will be reduced.

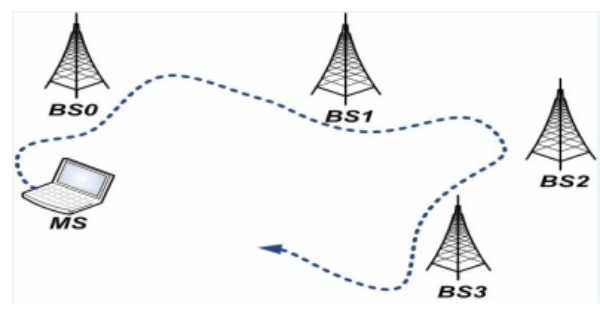

Fig. 4. FCS Scanning Scenario 
The MS scans the neighboring BSs based on the channel information provided by the serving BS. Once scanning is complete, the serving BS performs negotiation with the target BSs through a backbone message, and selects a single target BS that meets the bandwidth requirements requested by the MS. While the MS scans, data cannot be transmitted, causing data buffering and undermining the system's performance. Also we have to take care of the MS mobility factor in account. So, adaptive channel scanning [13] technique also is taken into consideration. The proposed scheme involves eliminating unnecessary BSs by performing negotiations with neighboring BSs prior to scanning. Through the backbone message, a BS sends information to neighboring BSs regarding the bandwidth required by the MS before the MS scans. A response from neighboring BSs allows the protocol to assess the MS's expected performance if the service is provided by the corresponding BS. Then the MS scans only the BSs that satisfy its requirements. This approach reduces the number of BSs to be scanned and the time required for scanning, ultimately reducing system interruptions. BS0 is the serving $\mathrm{BS}$, and $\mathrm{BS} 1, \mathrm{BS} 2$ and $\mathrm{BS} 3$ are neighboring BSs. Whereas BS1 and BS2 satisfy the MS' bandwidth requirements; BS3 cannot provide the necessary bandwidth. Target BS selection takes place in the order of negotiation scanning; BS3 is eliminated during the negotiation process, and therefore is no longer subjected to scanning. Scanning is performed only on BS1 and BS2. Upon receipt of the MOB_SCN-REQ message, BS0 transmits an HO pre-notification to BS1, BS2 and BS3 to notify them of the MS' ID, as well as its required bandwidth. The HO prenotification response informs BS0 that although BS1 and BS2 can provide the bandwidth that the MS requires, BS3 can only offer a lower bandwidth. The MS then receives the MOB_SCN-RSP message containing this data, and scans only BS1 and $\mathrm{BS} 2$, but not BS3. Based on the values obtained from scanning, BS1 or BS2 is selected as the target BS. The known procedure involves scanning every neighboring $\mathrm{BS}$, which decreases the system's performance due to suspended data transmission during the scanning process. On the other hand, the negotiation process takes place through the backbone network, and does not interrupt data transmission. Therefore, negotiation is performed prior to scanning, to identify the BSs that satisfy the MS requirements. Only the selected BSs are scanned, decreasing the number of scans. This ultimately reduces the suspension of data transmission.

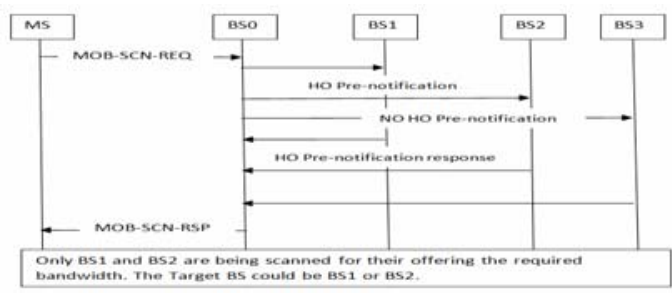

Fig. 5. FCS Scheme

\subsection{Traffic Manager (TM)}

Our second proposal's scenario is shown in figure 6, related to critical region applications that require QoS to ensure the bandwidth and delay requirements in case of high network loads. 
The main idea is that when a MS is transmitting a VoIP application through the assigned channel to its serving BS, and this channel capacity is over loaded, therefore, this MS will try to find an alternative path to forward its additional application streams. This dilemma use case led to the development of our Traffic Manager (TM). Traffic Manager receives bandwidth requests from the applications (supporting different services that require QoS), and uses a real-time view of the network topology and link capacities to calculate the best path for that connection. On this path, the demanded bandwidth is reserved for the lifetime of the connection. The MS must have two interfaces at least. Two data streams pass by each other; they may occupy the same space and bandwidth. To avoid flow interference each data stream should use different channel, here will require channel assignment issue or use one of the interfaces for one flow and the second interface for the other flow with a condition that the two interfaces are using different channels not a single one. This means that the first connections between the MS and the serving BS will be reserved over this path, without disconnecting it, and when this is congested, other request will be routed along other paths through the network. As a result, the network is able to adjust to dynamically changing traffic demands of the applications, guaranteeing the demanded bandwidth, and supporting a higher possible throughput between two WiMAX end points.

In addition to WiMAX PHY/MAC layers, there are three more functional entities essential to support Traffic Manager Server over WiMAX. The first one is a correct central real-time view of the network topology, and an accurate estimation of the bandwidth capacity of every channel between end points. The second one is a central software module that can calculate the path and possible bandwidth for every available neighbor BS for a bandwidth request within the network coverage. The third one is a mechanism to ensure that a connection does not consume more bandwidth then it has been assigned.

MS is transmitting a VoIP application through the first interface channel to its serving BS, and this channel capacity is facing traffic congestion issue resulted in the application degradation. Therefore, MS will try to find an alternative path to forward its additional application streams. MS will start an association context with Traffic Manager.

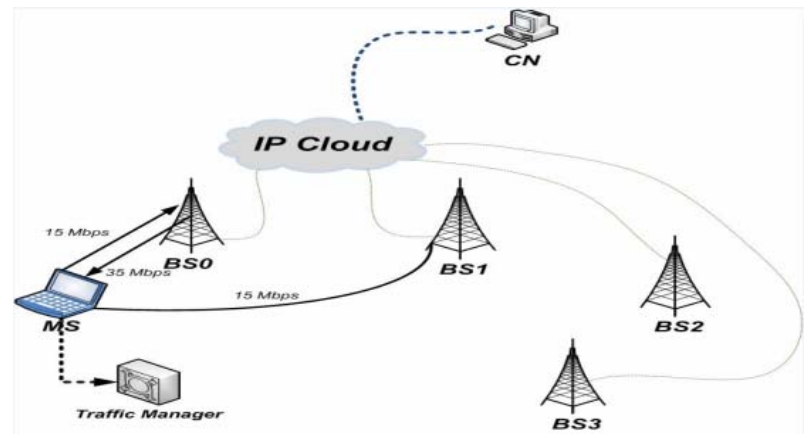

Fig. 6. TM Scenario 
The MS first interface (IF1) informs the TM of the bandwidth rate whenever an ACK frame is received or the bandwidth rate reaches the Bandwidth-limit. After recording the bandwidth rate in BAN1, the TM compares BAN1 with the Bandwidthlimit which is the threshold for switching to another network interface. In the case that BAN1 less than the Bandwidth-limit, the TM detects the deterioration of the condition of the wireless link and switches to the Two-path transmission in order to prevent packet loss and transmit the data packets on both interfaces.

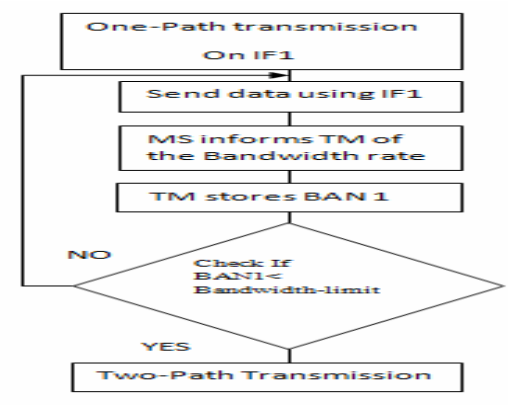

Fig. 7. Transmission to another Interface

In the Two-path transmission, since the MS sends data packets to the $\mathrm{CN}$ through both WiMAX interfaces, the networks load increases. When switching to the Twopath transmission, the bandwidth rate that a packet experiences is used as a switching criterion. The MS second WiMAX interface is assigned to communicate with the TM. In this case, there is a need for network load balancing in order not to decrease the connection capability, which is not considered in this study.

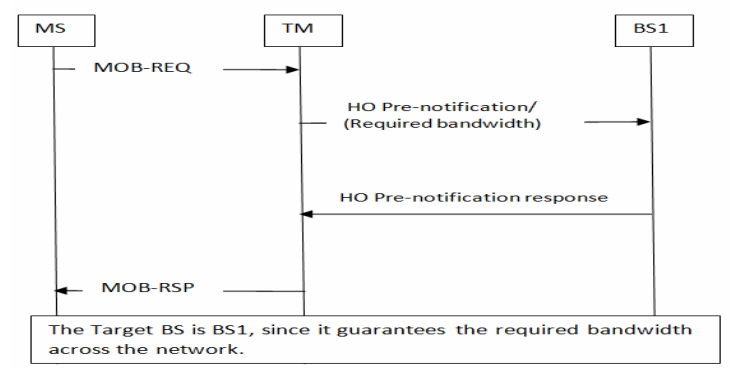

Fig. 8. TM Scheme

From the first proposal (FCS), we have gained a list of the best BSs estimated to offer the required bandwidth for data streams. In order to efficiently allocate UL bandwidth, UL scheduling specifies several types of services allow different levels of flexibility and efficiency carrying out the QoS parameters [9], Real-time Polling Service (rtPS) is selected in the parameter set. rtPS supports real-time data streams that generate variable size SDUs on a periodic basis, such as MPEG video. According to 
the UL bursts size that meets the flow's real-time needs, it allows the MS to specify the size of the desired grant. Therefore during the granted UL allocation, the MS sends PDUs containing the bandwidth request information. This service type requires more signalling overhead among the others, but supports flexible sizes for efficient data transport.Since the TM is aware of this list, it will forward the traffic to an alternative BS within the list. Here BS1 has been chosen to be the Target BS to communicate with the $\mathrm{CN}$.

TM server consists of an important entity (its structure is built in OPNET network modeller [12]) that is responsible for mapping video channel ID to Connection ID (CID), transmitting TM-MAC-PDU over WiMAX PHY/MAC, burst scheduling and allocating OFDMA data region for each PDU. Mapping video channels to multicast CIDs shall be known to all BSs belonging to the same TM server geographical zone.

WiMAX PHY/MAC at BS side applies PHY channel coding (specified by TM server) to each TM-MAC-PDU and maps each TM-MAC-PDU to the corresponding OFDMA data region slots (determined by TM server) for transmission. One TMDATA-IE for each TM-MAC-PDU will be transmitted in TM-MAP (at the beginning of TM zone) to indicate the CID and OFDMA data region.

At the MS side, according to video channel selected by a video channel selector [11] indicates WiMAX PHY/MAC to decodes only the TM-MAC-PDUs associated with the corresponding video channel (which translates into CID), thus saving power and bandwidth consumption. Communication entity between TM server and BS(s) is provided for transporting video packet from TM server to multiple BSs, which are physically separated in Multi-BS TM system.

When the MS intends to switch to another video channel as the available channel is congested, its TM server first gets the corresponding new Multicast CID via mapping from video channel to multicast CID. Then WiMAX PHY/MAC checks the TM-MAP in the new OFDMA frame locates the TM-DATA-IE containing the new multicast CID and starts decoding the corresponding TM-MAC-PDU. In the meantime, WiMAX PHY/MAC may stop decoding the TM-MAC-PDUs belonging to the congested video channel. Via TM-MAP redirection, WiMAX PHY/MAC knows the frame number of next frame containing interested TM-MAC-PDUs. Since TM server can locate and decode only the packets on the video channel currently being watched, no power will be wasted for decoding unwanted video packets. This may optimize the power consumption issue if required.

This proposal has the following features. First, we provide broadcast synchronization among multi-BSs through the cooperation between TM server and BSs. In other words, same video content will be transmitted at the same time and in same bandwidth across multi-BSs. In this way, a seamless handoff can be achieved from one cell to another in a feasible way.

\section{Simulation Model}

The OPNET v14.0 simulation tool [12]; is used in order to implement the scenarios shown in figure 4 and 6 . OPNET provides flexibility through enabling the design of new communication protocols and devices, which may be used along with a readily available library that has been developed. For the first scheme, we build our simulations 
on a simplified but typical scenario; for the physical layer specification, we assume OFDMA/TDD is used, the OFDMA frame duration $(F t)$ is $5 \mathrm{~ms}$, and the minimum OFDMA slot rate is $122 \mathrm{kps}$. We rely on a network topology which contains a serving BS and three neighboring BSs. The MS is aware of the neighboring BS list from neighbor advertisements before the HO. During scanning, the MS gets synchronized with 2 neighbors and finally chooses one after several ranging (according to the best bandwidth information) transactions.

Our second scenario; the mobile station has two WiMAX interfaces. MS is communicating with the correspondent node $(\mathrm{CN})$ through IF1 to BS1, connected the wireless network to IP cloud which is used here to represent the internet backbone connectivity. The MS' second interface is connected to the Traffic Manager server. Scalable Video coding (SVC) [14] is the codec used in the server which is an extended version of MPEG-4 H.264/AVC (for Advanced Video Coding) where the video quality degradation is smooth when users move within and across cells. SVC is a video coding technology that encodes the video at the highest resolution and allows the data bit-stream to be adapted to provide various lower resolutions streams, results in several types of encoded data streams with a consideration to their bandwidth requirement.

The one-way delay to the $\mathrm{CN}$ from each WiMAX BS network is varied: that from BS0 is set to $35 \mathrm{~ms}$ and that from BS1 is set to $10 \mathrm{~ms}$. Note the hidden Nodes issue is not considered in this study.

Two video streams are requested between MS and the serving BS in each direction, for UL 30 and DL $35 \mathrm{Mb} / \mathrm{s}$ respectively. Because the direct link between MS and BS has a capacity of $50 \mathrm{Mb} / \mathrm{s}$, the first stream is forwarded via this link. But the second stream needs another $15 \mathrm{Mb} / \mathrm{s}$, meaning that if the stream was forwarded over the same path, the link would be loaded with $65 \mathrm{Mb} / \mathrm{s}$, which is too much. Therefore, the Traffic Manager ensures that the second stream will be forwarded from MS to BS1at this time, to the corresponding node $(\mathrm{CN})$, enabling the streaming of both video sources from MS to CN. Since the SVC coding mechanism is being used as this will give the priority to the most important data streams to pass through the best channel. Therefore, the first stream is assigned to provide basic video quality with low bit-rate that is required by most users, and the second stream will pass the higher resolution data which is less important and can be removed when the available bandwidth is not sufficient.

As Synchronization of MS across multi-BS is hard to achieve since the same application i.e., video stream content has to be transmitted in the same OFDMA frame and by the same channel usage, the MS must try at least more than 1 OFDMA frame duration $(F t)$ in $T$ slot to get synchronized before ranging. Therefore assuming it take the MS $\left(\mu\left(0, T s y n c \_M a x\right)\right.$ and Tsync_Max is $\left(2 T^{*} F t\right)$ according to the initial synchronization and downlink quality estimations. If the synchronization is not successful after Tsync_Max, the neighboring BS is assumed invalid within the BS obtained list and no further actions are taken. So the Synchronization latency will be reduced:

$$
T \text { sync }(\text { proposed })=T \text { syncs } * \frac{N-S}{N} \text {. }
$$

As the Tsyncs is the standard Synchronization latency.

$N$ : number of BSs, $S$ : number of invalid BSs. 
The second part is the ranging transaction duration based on the bandwidth information: the MS must estimate an initial ranging request $(B)$. We assume the rate of this estimation follows a normal threshold limit $N(\operatorname{Bmin}, \delta) . \delta$ is the variance which is assumed as one bandwidth rate adjustment step. Bmin is the lower limit for the successful ranging bandwidth request. In this model, if and only if $B \min >B$, do the neighboring $\mathrm{BS}$ receive ranging requests. The MS must increase $B$ by 1 step and retry until $B \geq B$ min as it's done in the two path algorithm. In the optimized HO, the MS directly ranges with valid BSs that provide the required bandwidth. The total ranging transaction latency can be calculated as:

$$
\sum_{i}^{N} \sum_{j}^{M} \operatorname{Trang}_{(i, j)}
$$

While $N$ here is the number of valid neighboring BSs and $M$ represents the number of ranging retrials for the $i$ neighboring BS. In the seamless $\mathrm{HO}$, the MS need not wait for ranging responses from the neighboring BS. Therefore, the ranging latency is $M^{*}$ Trang_REQ, while Trang_REQ is the ranging request transmission delay $(F t)$. The RNG_RSP transporting latency through the backbone does not affect the ranging transaction latency since the MS is still served by the serving BS during ranging transactions. So the Scanning latency will be:

$$
\sum_{i}^{N} \operatorname{Tscan}_{(i)}=N *\left(T_{\text {sync }(\text { proposed })}+\sum_{j}^{M} \operatorname{Trang}_{(j)}\right) .
$$

The network re-entry delay in the optimized scheme Tent is the capability negotiation, authentication and registration plus a random handling duration is assumed to be $110 \mathrm{~ms}$ in heavy load. The assumed length of the Transmit Transition Gaps (TTG) is $5 \mathrm{~ms}$ which is small according to the forwarding algorithm in TM. Transmit Transition Gap (TTG), is the gap between the last sample of the downlink burst and the first sample of the subsequent uplink burst in a time division duplex (TDD) transceiver. This gap allows time for the base stations (BS) to switch from transmit to receive mode. During this gap, the BS is not transmitting modulated data but simply allowing the BS transmitter carrier to ramp down.This latency is eliminated in the optimized scheme since the MS still keeps the communication with the severing BS during the network re-entry process. As the result, HO latency of the optimised schemes can be calculated as:

$$
\left.T_{\text {handover }}=\sum_{i}^{N} T \text { scan }+(\text { Tsync_Max } * N * \text { Tent })\right) .
$$

\section{Results and Discussion}

We run the OPNET simulation to analysis effects of different parameters on the $\mathrm{HO}$ latency for both scenarios respectively.

The delay is a function of handover algorithm and traffic characteristics. FCS resulted in less delay as shown in figure below. 


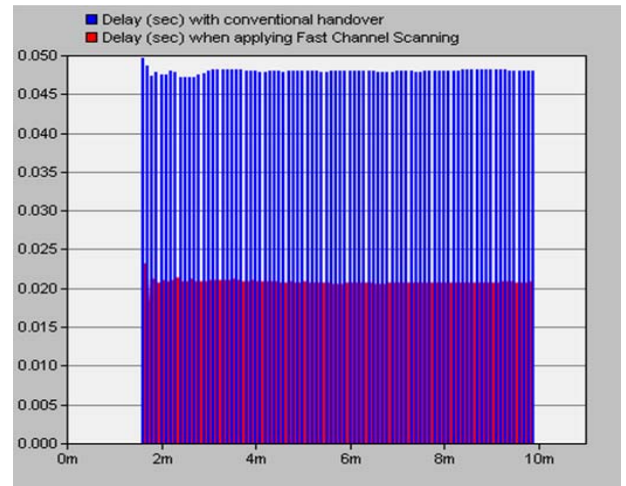

Fig. 9. The Handover Delay

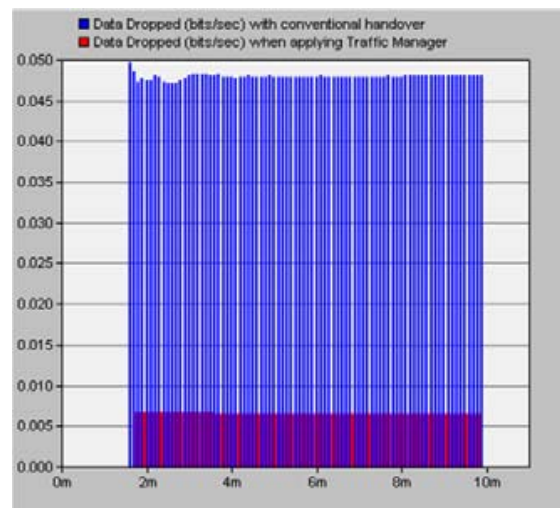

Fig. 10. Data Dropped During Handover

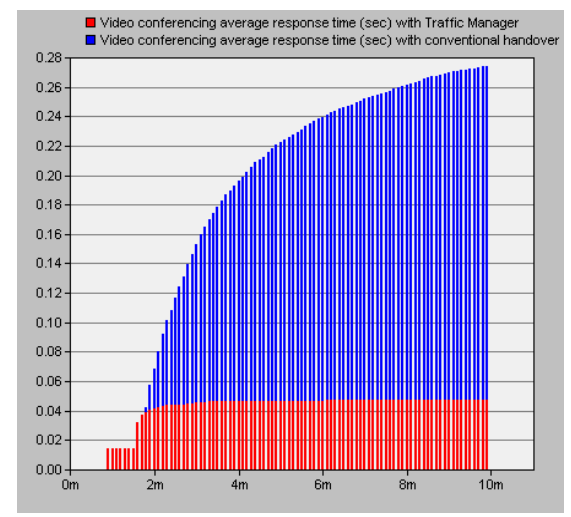

Fig. 12. Video Conferencing Average Response Time

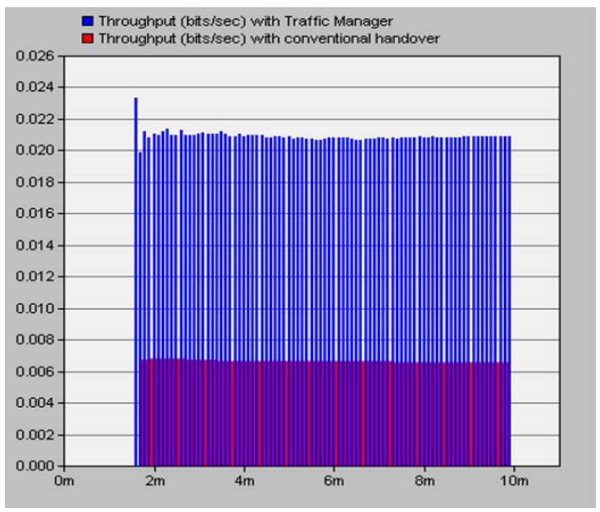

Fig. 11. Throughput performance

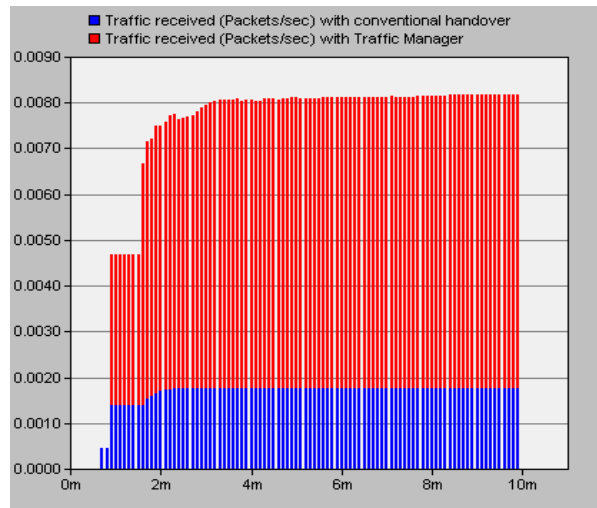

Fig. 13. Traffic Received 
The analysis results show that the FCS scheme as compared to the known conventional scheme can reduce the scan time, therefore handover latency up to $50 \%$ in the network topology acquisition process especially in association modes and successfully keeps handover delay under $30 \mathrm{~ms}$. The reduction comes from elimination of network unnecessary BSs by performing negotiations with neighboring BSs prior to scanning. Only selected BSs will be scanned and ranged within the network. This ultimately reduces number of scans, thus the suspension of data transmission

From figures 10,11, 12 and 13, it can be seen that the TM scheme can improve the video heavy load application's throughput in the network to more than 55\% (This statistic represents the average number of packets successfully received or transmitted by the receiver or transmitter channel per second), reduces the data dropped to $70 \%$, with assurance of traffic received to more than $60 \%$ by the target node (This statistic represents the total number of data packets received per second by target node from the network, across all interfaces).

It can be seen that the proposed handover algorithm improved the system efficiency and enhanced the overall network performance enormously in terms of fast application response time, of Video Conferencing applications in response time are $65 \%(20.766 \mathrm{sec})$ in $10 \mathrm{~ms}$ respectively as compared to conventional handover technique for WiMAX integrated networks.

The advantage of our cross-layer scheme is that the Performance does not degrade when the load increases, as the load will be distributed across different channels. In a word, these fast handover schemes can successfully reduce the waste of wireless resources and improve the performance of IEEE 802.16 broadband wireless networks and its mobility requirement during the handover process.

\section{Conclusion}

In this paper, we have proposed solutions to select a better base station and initiating handover to that station. The aim of the (FCS) Fast Channel Scanning is to reduce the scanning time pre- handover by elimination the number of BSs to be scanned according to the required bandwidth support, providing a clear evidence of how it successfully improves the mobile WiMAX handover and addresses the latency issue. The second proposal Traffic Manager (TM), when a mobile station detects that the current channel quality of the primary path becoming degraded as a result of traffic congestion on that channel, the mode is switched to another transmission interface according to the bandwidth condition. Through simulations, we have shown several performances at the time the MS switches to different network to adapt the channel congestion problem and how it severely restricts the amount of critical video streams, introducing an algorithm that based on the channel bandwidth information, which effectively reduce the mobile WiMAX handover (HO) latency. Future work, will aim to convert the TM server scheme to a protocol design that will add more applications in addition to video traffic in large mesh networks, to improve the system scalability and other capacity issues of the network. Since the proposed traffic management scheme had MS equipped with two WiMAX interfaces that is impractical as it will increase the hardware cost of MSs If an intention to bring this product/service to market. 


\section{References}

1. IEEE Std 802.16-2004, IEEE Standard for Local and Metropolitan Area Networks, Part 16: Air Interface for Fixed Broadband Wireless Access Systems

2. IEEE Std 802.16e-2005, IEEE Standard for Local and Metropolitan Area Networks, Part 16: Air Interface for Fixed Broadband Wireless Access Systems, Amendment 2: Physical and Medium Access Control Layers for Combined Fixed and Mobile Operation in Licensed Bands, Corrigendum 1

3. Etemad, K.: Overview of mobile WiMAX technology and evolution. IEEE Communications Magazine (October 2008)

4. Choi, S., Hwang, G.-H., Kwon, T., Lim, A.-R., Cho, D.-H.: Fast Handover Scheme for Real-Time Downlink Services in IEEE 802.16e BWA System. In: Vehicular Technology Conference (2005 IEEE 61st), vol. 3, pp. 2028-2032 (2005)

5. Speicher, S., Bünnig, C.: Fast MAC-Layer Scanning in IEEE 802.11 Fixed Relay Radio Access Networks. In: ICN/ICONS/MCL, April 23-29 (2006)

6. Kyung-ah, K., Chong-Kwon, K., Tongsok, K.: A seamless handover Mechanism for IEEE 802.16e Broadband Wireless Access. In: ISPC 2004 (2004)

7. Chen, L., Cai, X., Sofia, R., Huang, Z.: A Cross layer Fast Handover Scheme For Mobile WiMAX. IEEE Press, Los Alamitos (2007)

8. Li, B.: Hong Kong University of Science and Technology Yang Qin and Chor Ping Low, Nanyang Technological University Choon Lim Gwee, Republic Polytechnic: A Survey on Mobile WiMAX. IEEE Communications Magazine (December 2007)

9. Walke, B.H., Mangold, S., Berlemann, L.: IEEE 802 Wireless Systems. John Wiley and Sons Ltd., Chichester (2006)

10. Hu, R.Q., Paranchych, D., Fong, M.-H., Wu, G.: On the Evolution of Handoff Management and Network Architecture in WiMAX. IEEE, Los Alamitos (2007)

11. Optimized Network Engineering Tools, OPNET Technologies, session 1827 (2007), http: / / www . opnet. com

12. Optimized Network Engineering Tools (2008), http: / / www . opnet. com

13. Rouil, R., Golmie, N.: Adaptive Channel Scanning for IEEE 802.16e. In: Proceedings of 25th Annual Military Communications Conference (MILCOM 2006), Washington, D.C., October 23-25 (2006)

14. Schwarz, H., Marpe, D. (Member, IEEE), Wiegand, T., (Member, IEEE): Overview of the Scalable Video Coding Extension of the H.264/AVC Standard. IEEE transactions on circuits and systems for video technology 17(9) (September 2007) 